\title{
A Novel Technique for Fetal Heart Rate Estimation Based on Ensemble Learning
}

\author{
Lu Zhang $^{1} \&$ Mei-Jia Huang ${ }^{1} \&$ Hui-Jin Wang ${ }^{1}$ \\ ${ }^{1}$ School of Information Science and Technology, Jinan University, Guangzhou, China \\ Correspondence: Hui-Jin Wang, School of Information and Technology, Jinan University, Guangzhou, China. \\ E-mail: twanghj@jnu.edu.cn
}

Received: September 2, 2019

Accepted: September 25, 2019

Online Published: September 27, 2019

doi:10.5539/mas.v13n10p137

URL: https://doi.org/10.5539/mas.v13n10p137

\begin{abstract}
The autocorrelation algorithm is the most commonly used method for extracting fetal heart rate from ultrasound Doppler fetal monitors. The traditional autocorrelation algorithm can not always extract the detection cycle accurately. During the calculation process, the heartbeat cycle may not be recognized, or the cycle may be doubled or halved recognized. Combining the characteristics of envelope curve with average magnitude difference function curve, this paper designs a set of extreme point search scheme and a fetal heart cycle recognition model based on ensemble learning to assist in screening the best fetal heart cycle. The aim of this study is to improve the precision of the fetal heart rate calculation. The experimental results show that the proposed method can effectively screen out the best fetal heart cycle with enhanced reliability and robustness.
\end{abstract}

Keywords: autocorrelation algorithm, ensemble learning, fetal heart rate(FHR), average magnitude difference function

\section{Introduction}

During pregnancy, fetal heart rate (FHR) monitoring is an essential routine examination, which is of great significance to reduce the fetal mortality rate and guarantee the health and safety of the fetus (Xiao, Luo \& Zhang, 2005).

Doppler Ultrasound Signal (DUS) is the most diffused non-invasive measurement in FHR monitoring; however, it is susceptible to the abdominal murmur of the pregnant mother that results in signal loss and misdiagnosis. The autocorrelation function(ACF) is commonly used in existing fetal monitors (M. Peters et al., 2001) to improve this situation. As the period of the ACF is consistent with the input ultrasonic Doppler fetal heart sound signal, the heartbeat period of the FHR can be obtained by calculating the period of the ACF and the fetal heart rate can be obtained through the calculation.

Based on traditional ACF, many domestic and foreign scholars have done much work and proposed many effective detection algorithms. C. Peters et al. (2004) proposed an algorithm for calculating FHR by beat, and the accuracy is close to which is obtained directly from FECG. Lee et al. (2009) improved noise immunity based on Peters' algorithm to achieve higher accuracy and robustness. Voicu et al. (2010) used an ultrasonic multi-channel Doppler system by modifying ACF and proposed a new time and frequency estimator for the audio domain, and thus the miss rate of FHR has reduced to 5\%. Jezewski et al. (2011) achieved a high accuracy of fetal cardiac intervals measurement by adopting dynamic adjustment of autocorrelation window, adaptive autocorrelation peak detection and the determination method of the beat-to-beat interval, making it possible to reliably and quantitatively evaluate the FHR variability. Marzbanrad et al. (2014) proposed an automated estimation of fetal cardiac intervals from a DUS signal based upon a unique combination of empirical mode decomposition (EMD) and hybrid support vector machine-hidden Markov model(SVM/HMM). Al-Angari et al. (2017) proposed a new method based on EMD and kurtosis statistics to estimate fetal heart rate and variability of fetal Doppler ultrasound signal, showing excellent performance in estimating fetal beat to beat interval. Recently, Seong (2018) has disclosed a method for extracting fetal heart rate in, in which average magnitude difference function (AMDF) and ACF are used.

Ensemble learning is a machine learning paradigm where multiple models are trained to solve the same problem and combined to get better results, that means when weak models are correctly combined we can obtain more 
accurate and robust models.

In this study, ensemble learning techniques were applied in fetal cardiac interval recognition and ten features are extracted from envelope curve and average magnitude difference function (AMDF) as the input to classify the fetal cardiac interval into right and wrong. It is shown that good classification accuracy can be achieved by using this novel method.

The remainder of this paper is organized as follows: In Section2, the preprocessing of database, feature selection and the new approach based on ensemble learning is proposed. The experimental result is summarized in Section3. In Section 4, the conclusion of this research is presented.

\section{Method}

This paper proposes a fetal cardiac interval recognition model based on ensemble learning to assist in identifying fetal heart rate. Features extracted from envelope curve and average magnitude difference function (AMDF) curve are used to judge whether the current cycle points can obtain the correct fetal heart rate.

\subsection{Acquisition and Preprocessing}

In this paper, the database is provided by Guangzhou Overseas Chinese Hospital. Thirty-six valid original Doppler fetal heart sound signal are selected to construct a training database by following steps:

1) Down-sampling. The frequency of the original signal $S$ is $8000 \mathrm{HZ}$, and the sampling frequency is reduced to $250 \mathrm{HZ}$ by performing a sum operation every 32 points to reduce the complexity of the calculation. Then get the new signal $\boldsymbol{S}^{\prime}$. (Figure 1.a)

2) Get envelope signal. from the new signal $\boldsymbol{S}$ '. Calculate the mean value (Mean(Seg)) of signal $\boldsymbol{S}$ ' every 2 seconds (Seg). Obtain envelope signal $\boldsymbol{E}$ according to equation (1) and (2). (Figure 1.b)

$$
\begin{gathered}
\text { fix num }=\frac{\text { Mean }(\text { Seg })}{30} \\
E_{i}=\frac{\text { Seg }_{\mathrm{i}}}{\text { fixnum }^{-}}
\end{gathered}
$$

3) Get AMDF signal $\boldsymbol{C}$ from the envelope signal $\boldsymbol{E}$ obtained from step 2) by means of AMDF. (Figure 1.c) 


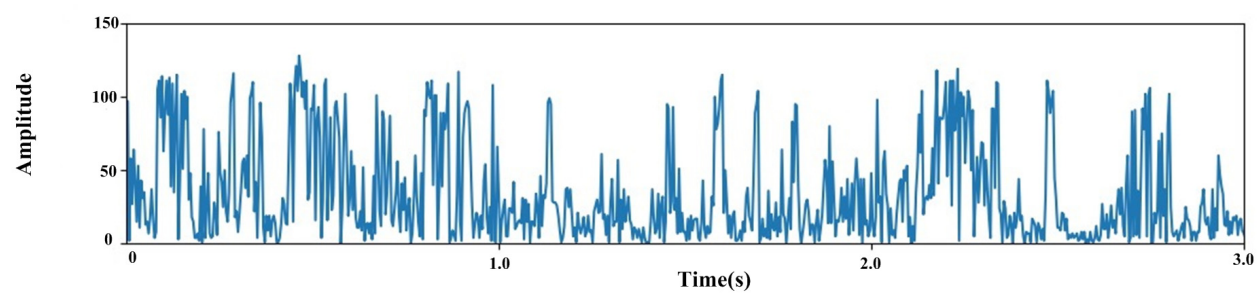

a) original signal

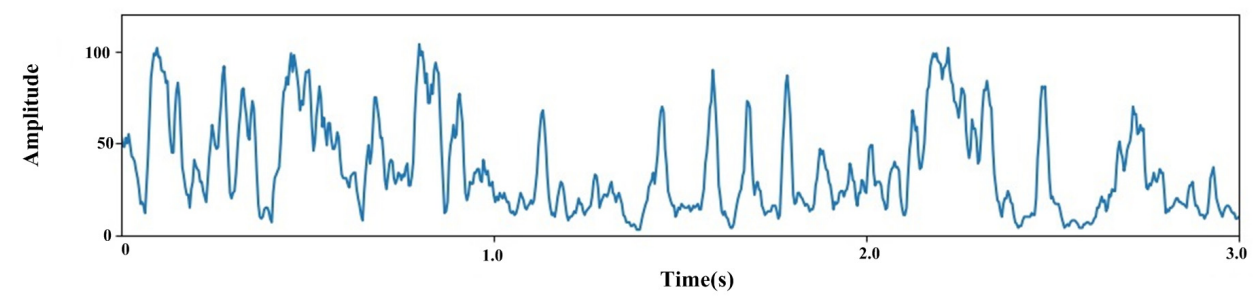

b) envelope signal

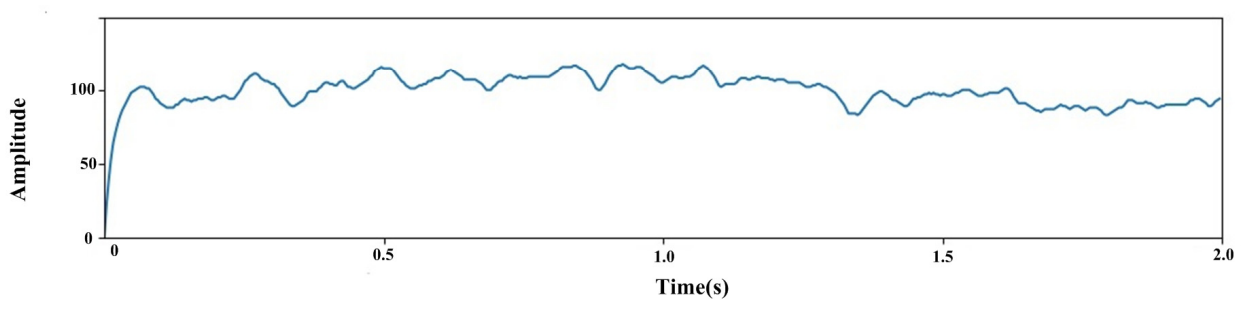

c) AMDF signal

Figure 1. An overview of a) original signal, b) envelope signal and c) AMDF signal

\subsection{Feature Extraction and Selection}

Extracting features is mainly based on the following characteristics of fetal heart rate:

1) The normal fetal heart rate is between $110 \mathrm{bpm}$ and $160 \mathrm{bpm}$ (W. Cheng \& Z. Cheng, 2018) (or between 120 bpm and $160 \mathrm{bpm}$ (Xiao, Luo \& Zhang, 2005)), and most of the fetal heart rate is concentrated in this range, even in the case of poor signal. The measuring range of the electronic fetal monitor is from $50 \mathrm{bpm}$ to $210 \mathrm{bpm}$ or $30 \mathrm{bpm}$ to $240 \mathrm{bpm}$ (W. Cheng \& Z. Cheng, 2018). If the calculated fetal heart rate exceeds the above measurement range, there is a large probability that it is a false fetal heart rate value.

2) Generally, dramatic mutations of the fetal heart rate do not occur, and the difference between adjacent heartbeat cycles is not more than $10 \%(\mathrm{Li}, 2005)$ Therefore, when the front and rear heart cycles are relatively close, it can be considered that the detected heartbeat cycle has a high credibility at this time.

\subsubsection{Select Features from AMDF Curve}

First, a simple method of searching for minimum points is adopted. The main steps are as follows:

1) Start from the AMDF curve $\boldsymbol{C}$, and search the minimum point $\boldsymbol{P}_{\boldsymbol{l}}$ in the range $[\boldsymbol{i}, \boldsymbol{i}+$ step $]$, where the step is 50 and the initial value of $\boldsymbol{i}$ is 0 .

2) Search the minimum point $\boldsymbol{P}_{2}$ in the range $[\boldsymbol{i}+$ step, $\boldsymbol{i}+2 *$ step $]$. If $\boldsymbol{P}_{\boldsymbol{l}}=\boldsymbol{P}_{2}$, then add the point $\boldsymbol{P}_{1}$ to the extreme point sequence $\boldsymbol{V}$, and set $\boldsymbol{i}=\boldsymbol{i}+\boldsymbol{2}$ *step. If $\boldsymbol{P}_{1} \neq \boldsymbol{P}_{2}$, set $\boldsymbol{i}=\boldsymbol{i}+\boldsymbol{1}$.

3) Continue the search with steps a-b until the end of the curve.

Set the time of the current calculated FHR in signal $\boldsymbol{S}^{\prime}$ as $\boldsymbol{T}_{\boldsymbol{c}}$, and the x-coordinate time value corresponding to the element $\boldsymbol{P}_{i}$ in the extreme point sequence $\boldsymbol{V}$ is $\boldsymbol{x}_{\boldsymbol{i}}$ (The subscript of the sequence $\boldsymbol{V}$ starts from 1). Extract the periodic time series $T=\left\{\boldsymbol{t}_{\boldsymbol{l}}, \boldsymbol{t}_{2}, \ldots, \boldsymbol{t}_{\boldsymbol{k}}\right\}$ from the sequence $\boldsymbol{V}$, which are shown in Figure $2 \mathrm{a}$. 


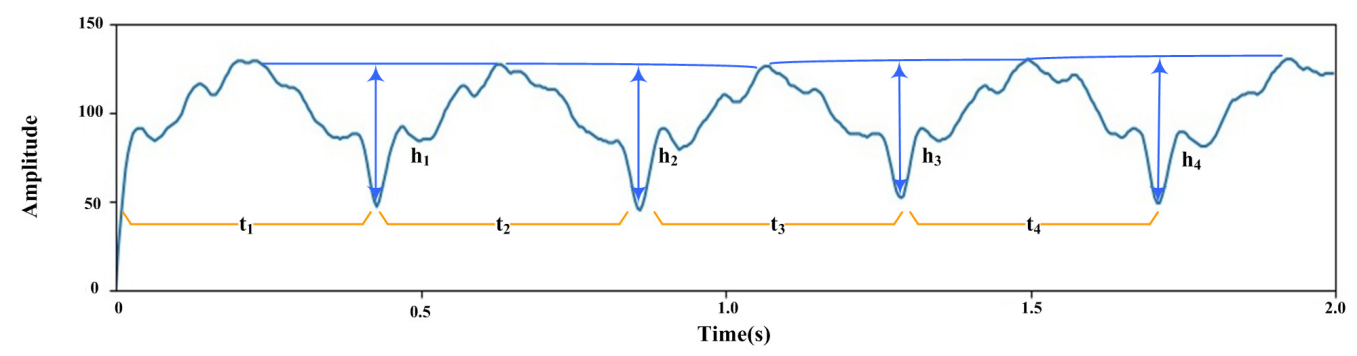

Figure 2. Characteristics of AMDF curve a) The yellow lines symbolize the periodic time of AMDF curve, b) The blue lines symbolize the amplitude difference of AMDF curve

At the same time, $\boldsymbol{x}_{i}$ will be selected as the pre-selected current fetal heartbeat cycle time ( $\boldsymbol{i}$ is initialized to 1), which will be converted into pre-selected fetal heart rate, according to equation:

$$
F H R_{c}=\frac{60}{x_{i}}
$$

$\boldsymbol{F}_{q}$ is used to measure the periodic strength of the AMDF curve. When each adjacent interval time satisfies $0.9 \leq$ $\boldsymbol{t}_{\boldsymbol{i}+1} / \boldsymbol{t}_{i} \leq 1.1$, the intensity value of $\boldsymbol{F}_{q}$ increases by 1 , and the larger it is, the clearer the period is.

For the item in $\boldsymbol{T}$ which increases the value $\boldsymbol{F}_{\boldsymbol{q}}$, take the average value $\boldsymbol{t}_{\text {mean }}$ and convert it into heart rate value $\boldsymbol{F H} \boldsymbol{R}_{\boldsymbol{m}}$ in the same way as formula (3).

$\boldsymbol{F}_{\boldsymbol{t}}$ is used to measure the periodic confidence of the extreme point in the AMDF curve. $\boldsymbol{F}_{\boldsymbol{t}}$ is calculated as follows:

$$
F_{t}=\left\{\begin{array}{l}
3, \text { if } 110<F H R_{m} \leq 160 \\
2, \text { if } 50<F H R_{m} \leq 110 \text { or } 160<F H R_{m} \leq 210 \\
1, \text { if } 30<F H R_{m} \leq 50 \text { or } 210<F H R_{m} \leq 240 \\
0, \text { else }
\end{array}\right.
$$

The magnitude between the minimum and the adjacent maximum points is extracted as a measurement index. Figure 2.b, shows the sequence of magnitude difference of the extreme points $\boldsymbol{H}=\left\{\boldsymbol{h}_{\boldsymbol{l}}, \boldsymbol{h}_{2}, \ldots, \boldsymbol{h}_{\boldsymbol{j}}\right\}$.

$\boldsymbol{K}_{\boldsymbol{h}}$, similarity coefficient, is used to measure the similarity between the magnitude difference of the pre-selected extreme points and the overall average magnitude difference. $\boldsymbol{K}_{\boldsymbol{h}}$ is calculated as:

$$
K_{h}=\frac{h_{i}}{\text { Mean }(H)}
$$

$\boldsymbol{F}_{\boldsymbol{h}}$ is used to assist in measuring the quality of the minimum point of AMDF curve, $\boldsymbol{F}_{\boldsymbol{h}}$ is calculated as follow ( $\boldsymbol{A} \boldsymbol{b} \boldsymbol{s}$ is an absolute value function):

$$
F_{\mathrm{h}}=\left\{\begin{array}{l}
3, \text { ifAbs }\left(1-K_{h}\right) \leq 0.1 \\
2, \text { if } 0.1<A b s\left(1-K_{h}\right) \leq 0.15 \\
1, \text { if } 0.15<A b s\left(1-K_{h}\right) \leq 0.5 \\
0, \text { else }
\end{array}\right.
$$

$\boldsymbol{F}_{\boldsymbol{s}}$ is used to measure the morphological characteristics of the pre-selected extreme point, $\boldsymbol{F}_{\boldsymbol{s}}$ is calculated as follow (Floor represents the round down function):

$$
F_{s}=\text { Floor }\left(0.25 \times \frac{h_{i}}{t_{i}}\right)
$$

$\boldsymbol{F}_{\boldsymbol{n}}$ is used to assess the credibility of the current pre-selected fetal heart rate. During the calculation process of fetal heart rate, the latest time of $\boldsymbol{T}_{r}$ and $\boldsymbol{F} \boldsymbol{H} \boldsymbol{R}_{r}$ will be recorded. In general, there will not be large mutations in adjacent time. $\boldsymbol{F}_{\boldsymbol{n}}$ is calculated as follow ( $\boldsymbol{A} \boldsymbol{b} \boldsymbol{s}$ is an absolute value function) : 


$$
F_{n}=\left\{\begin{array}{l}
3, \text { if } \frac{A b s\left(F H R_{r}-F H R_{c}\right)}{F H R_{r}} \leq 5 \% \text { and }{ }_{1}-t_{r} \leq 2 s \\
2, \text { if } 5 \%<\frac{A b s\left(F H R_{r}-F H R_{c}\right)}{F H R_{r}} \leq 10 \% \text { and }_{1}-t_{r} \leq 4 s \\
1, \text { if } 10 \%<\frac{A b s\left(F H R_{r}-F H R_{c}\right)}{F H R_{r}} \leq 20 \% \text { and }_{1}-t_{r} \leq 6 s \\
0, \text { else }
\end{array}\right.
$$

\subsubsection{Select Features from Envelope Curve}

For the sequence of envelope signal $\boldsymbol{E}, \boldsymbol{E}_{\boldsymbol{m}}$ is the mean value of the signal $\boldsymbol{E}$ in the corresponding time period and is extracted as an indicator to measure the quality of the signal.

$\boldsymbol{F}_{\boldsymbol{m}}{ }_{\boldsymbol{m}}$, is used to measure the quality of envelope signal. $\boldsymbol{F}_{\boldsymbol{m}}{ }_{\boldsymbol{m}}$ is calculated as follows:

$$
F_{\mathrm{h}}=\left\{\begin{array}{l}
2, i f v_{l}<E_{m}<v_{h} \\
1, i f E_{m}<v_{l} \\
0, E_{m}>v_{h}
\end{array}\right.
$$

Where $v_{1}$ and $v_{\boldsymbol{h}}$ are empirical coefficients, $\boldsymbol{v}_{1}=10, \boldsymbol{v}_{\boldsymbol{h}}=50$.

Search the maximum points of the envelope signal curve, use the same method as the AMDF curve used, and extract the time intervals between the maximum points, where the time interval sequence is $T^{\prime}=\left\{\hat{t}_{1}, t_{2}, \ldots, t_{k}\right\}$.

$\boldsymbol{F}_{q}^{\prime}$, is used to measure the periodic strength of envelope cycle. Considering that the extreme points of the envelope curve are likely to have non-periodic points under the search strategy, a filter is designed to filter the extreme points. The main steps are as follows:

1) Convert the time interval in $\boldsymbol{T}$ ' to the fetal heart rate value, and keep it when the fetal heart rate is between $50 \mathrm{bpm}$ and $210 \mathrm{bpm}$, otherwise, discard it. After preliminary filtration, a new sequence $\boldsymbol{T}_{\text {normal }}^{\prime}$ is obtained.

2) For the new sequence $\boldsymbol{T}_{\text {normal }}$, the number of elements in $\boldsymbol{T}_{\text {normal }}$ is counted every $10 \mathrm{bpm}$ in the range of 50 $\mathrm{bpm}-210 \mathrm{bpm}$. Finally, the element with the highest frequency is retained to obtain the new sequence $\boldsymbol{T}_{\text {select }}^{\prime} \boldsymbol{F}_{q}^{\prime}$ is calculated as follows (Count is the counting function):

$$
F_{\mathrm{q}}^{\prime}=\left\{\begin{array}{l}
2, \text { if } 2<\text { Count }\left(T_{\text {select }}^{\prime}\right) \leq 5 \\
1, \text { if } 1 \leq \text { Count }\left(T_{\text {select }}^{\prime}\right) \leq 2 \\
0, \text { else }
\end{array}\right.
$$

Then take the average value of $\boldsymbol{T}_{\text {select }}$ as $\boldsymbol{M e a n}\left(\boldsymbol{T}_{\text {select }}\right.$, and convert it to $\boldsymbol{F H \boldsymbol { R } _ { \boldsymbol { m } }}$ according to the formula (3). Also, the first time period interval in $\boldsymbol{T}_{\text {select }}$ is converted to $\boldsymbol{F} \boldsymbol{H} \boldsymbol{R}_{\boldsymbol{t}}^{\prime}$ as the pre-selected fetal heart rate of $\boldsymbol{E}$.

$\boldsymbol{F}_{\boldsymbol{t}}{ }^{\prime}$ is used to measure the credibility of the period of envelope, and similar to the calculation of $\boldsymbol{F}_{\boldsymbol{t}}$ in the AMDF curve. $\boldsymbol{F}_{\boldsymbol{t}}$ is calculated as follow:

$$
F_{t}{ }^{\prime}=\left\{\begin{array}{l}
3, \text { if } 110<F H R_{m} \leq 160 \\
2, \text { if } 50<F H R_{m} \leq 110 \text { or } 160<F H R_{m} \leq 210 \\
1, \text { if } 30<F H R_{m} \leq 50 \text { or } 210<F H R_{m} \leq 240 \\
0, \text { else }
\end{array}\right.
$$

$\boldsymbol{F}_{n}^{\prime}$ is used to assess the credibility of current $\boldsymbol{F H} \boldsymbol{R}_{\boldsymbol{t} \boldsymbol{1}} . \boldsymbol{F}_{\boldsymbol{n}}$ is calculated as follow:

$$
F_{n}^{\prime}=\left\{\begin{array}{l}
3, \text { if } \frac{A b s\left(F H R_{r}-F H R_{t_{1}}^{\prime}\right)}{F H R_{r}} \leq 5 \% \text { and }{ }_{1}-t_{r} \leq 2 s \\
2, \text { if } 5 \%<\frac{A b s\left(F H R_{r}-F H R_{t_{1}}^{\prime}\right)}{F H R_{r}} \leq 10 \% \text { andt }{ }_{1}-t_{r} \leq 4 s \\
1, \text { if } 10 \%<\frac{A b s_{r}\left(F H R_{r}-F H R_{t_{1}}^{\prime}\right)}{F H R_{r}} \leq 20 \% \text { and }{ }_{1}-t_{r} \leq 6 s \\
0, \text { else }
\end{array}\right.
$$

$\boldsymbol{K}_{e}^{\prime}$, similarity coefficient, is used to assess the similarity between $\boldsymbol{F H} \boldsymbol{R}_{\boldsymbol{t}}^{\prime}$ and $\boldsymbol{F H} \boldsymbol{R}_{\boldsymbol{m}}$, The greater the similarity is, the higher confidence of the fetal heart rate becomes. 


$$
K_{e}=\frac{A b s\left(F H R_{t_{1}}^{\prime}-F H R_{m}^{\prime}\right)}{F H R_{t_{1}}^{\prime}}
$$

Fe is used to assist in measuring the credibility of current FHR't1, Fe is calculated as follow:

$$
F_{e}=\left\{\begin{array}{l}
2, \text { if } K_{e}<0.05 \\
1, \text { if } 0.05 \leq K_{e}<0.1 \\
0, \text { else }
\end{array}\right.
$$

Finally, ten features were extracted from Doppler fetal heart sound signal. In addition, there are two signal marker features $\boldsymbol{T a g}_{1}$ and $\boldsymbol{T a g}_{2}$. If $\boldsymbol{T a g}_{\boldsymbol{1}}=1$, it means that the AMDF curve signal were selected as the main extracted signal; if $\boldsymbol{T a g}_{2}=1$, it means that the envelope curve signal was selected. The final extracted features are shown in Table 1.

\begin{tabular}{|c|c|c|c|}
\hline Source of feature & Feature symbol & Remarks & $\begin{array}{c}\text { Equatio } \\
n\end{array}$ \\
\hline \multirow{6}{*}{ AMDF curve } & $\begin{array}{c}F_{q} \\
F_{t}\end{array}$ & $\begin{array}{l}\text { Measure the period strength of the AMDF curve } \\
\text { Measure the credibility of the AMDF extreme } \\
\text { points }\end{array}$ & $\overline{(4)}$ \\
\hline & $F_{h}$ & $\begin{array}{l}\text { Measure the quality of the minimum point of the } \\
\text { AMDF curve }\end{array}$ & (6) \\
\hline & $F_{s}$ & $\begin{array}{l}\text { Measure the positional morphological } \\
\text { characteristics }\end{array}$ & (7) \\
\hline & & of pre-selected extreme points & \\
\hline & $F_{n}$ & $\begin{array}{l}\text { Assess the credibility of the current pre-selected } \\
\text { fetal heart rate }\end{array}$ & (8) \\
\hline & $\operatorname{Tag}_{1}$ & The flag of the signal is selected prioritized & - \\
\hline \multirow{6}{*}{ envelope curve } & $F_{m}^{\prime}$ & Measure the quality of signal & (9) \\
\hline & $F_{q}^{\prime}$ & Measure the periodic strength of envelope cycle & (10) \\
\hline & $F_{t}^{\prime}$ & $\begin{array}{l}\text { Measure the credibility of the period of envelope } \\
\text { curve }\end{array}$ & (11) \\
\hline & $F_{n}^{\prime}$ & Assess the credibility of current $\boldsymbol{F H} \boldsymbol{R}_{t \boldsymbol{I}}^{\prime}$ & (12) \\
\hline & $F_{e}$ & Assist in measuring the credibility of current $\boldsymbol{F} \boldsymbol{H} \boldsymbol{R}_{t \boldsymbol{t}}^{\prime}$ & (14) \\
\hline & $\mathrm{Tag}_{2}$ & The flag of the signal is selected prioritized & - \\
\hline
\end{tabular}

Table 1. Ten features extracted from the Doppler fetal heart sound signal

\subsection{Fetal Heart Cycle Recognition Model Based on Ensemble Learning}

This paper adopts the Stacking algorithm of ensemble learning, which is divided into two layers of learning algorithms. The basic level is mainly composed of KNN, SVM, MLP and Adaboost, which are trained based on a complete training set. The output obtained by each classifier of basic layer is as the input of XGBoost classifier (the second layer) to get the final classification result. 5-fold cross validation was used in the training process, and the data was split into 5 parts. For the training process, it is necessary to use four data elements while the testing process needs the residuary part to perform. To finish the process, the procedure was repeated 5 times to allow each fraction of data as a testing data. The whole training process of the model was shown in Figure 3. 


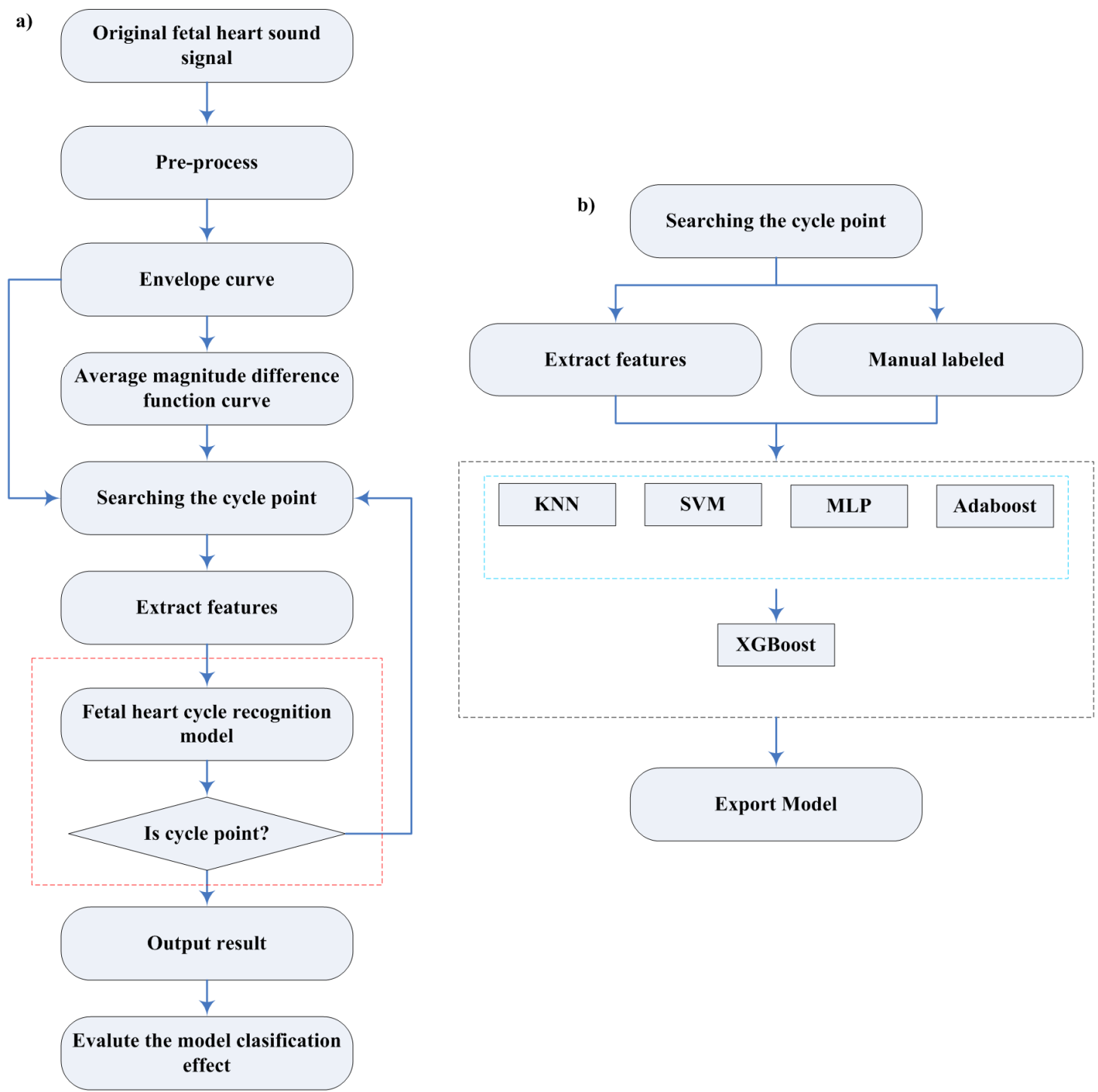

Figure 3. a) the complete process of fetal heart rate extraction model b) shows the detail process in the red box of a)

After training, evaluate the generalization ability of the model and integrate the model into the extraction process of fetal heart rate. First, new Doppler fetal heart sound signal data will be selected for preprocessing and feature extraction. Second, the pre-selected fetal heart rate obtained from the periodic sequence of AMDF curve and the periodic sequence of envelope curve combined with the extracted features are input into the model sequentially to predict the output. If the output is positive, it is considered that the fetal heart rate has been obtained at the current moment, then entering the process of fetal heart rate extraction at the next moment, or else it is considered that the current fetal heart rate value cannot be obtained, then to re-select the period points and go on. Finally, the fetal heart rate is compared with the correct result to evaluate the performance of the model.

\section{Results and Discussion}

In the collection of training samples, the proportion of negative samples is small. The upsampling algorithm SMOTE (Chawla et al., 2002) is used to balance the positive and negative ratio of training samples. 114,695 pieces training data and 1549 pieces test data were obtained, as shown in Table 2. 
Table 2. Basic information of dataSet

\begin{tabular}{ccc}
\hline Sample & Category & Number \\
\hline & Positive & 54241 \\
Training Set $\left(\mathrm{D}_{\text {train }}\right)$ & Negative & 60454 \\
& & \\
Testing Set $\left(\mathrm{D}_{\text {test }}\right)$ & Total number & 114695 \\
\hline
\end{tabular}

The numbers of true negatives (TN), false negative (FN), true positives (TP) and false positives (FP) are used to compute the efficiency of the classifier. Precision is the fraction of relevant instances among the retrieved instances.

$$
\text { Precision }=\frac{\mathrm{TP}}{\mathrm{TP}+\mathrm{FP}} \times 100 \%
$$

Recall is the fraction of the relevant instances that are successfully retrieved.

$$
\text { Recall }=\frac{\mathrm{TP}}{\mathrm{TP}+\mathrm{FN}} \times 100 \%
$$

There is another statistical measurement call F1-score, to evaluate characterization of the performance, which has the following formula:

$$
F 1=\frac{2 \times \text { Precision } \times \text { Recall }}{\text { Precision }+ \text { Recall }} \times 100 \%
$$

Figure 4 shows the comparison of algorithm performance in the ensemble algorithm. The performance of each machine learning method is good, and this demonstrates the effectiveness of feature extraction method and classifier, on the other hand, it also demonstrates that the SMOTE has improved the performance of model. Although the performance of each algorithm is relatively close, the ensemble algorithm gets the maximum performance of classification with the highest precision and F1-score.

The Table 3 shows the mean value of 5-fold cross validation of ensemble classifier. The precision is $99.53 \%$, the recall rate is $98.39 \%$, and the F1-score is $98.96 \%$. Therefore, we chose ensemble classifier to train the model.

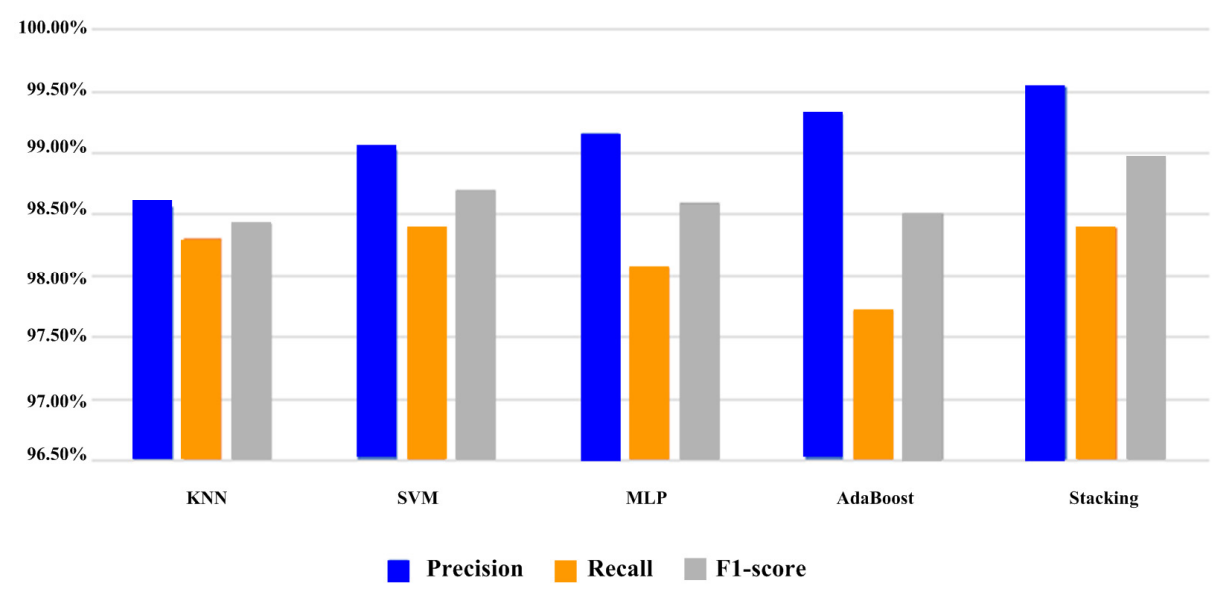

Figure 4. The performance of the respective machine learning methods 
Table 3. Result of the 5 -fold cross validation

\begin{tabular}{ccccccc}
\hline & 1 & 2 & 3 & 4 & 5 & mean \\
\hline Precision & $99.55 \%$ & $99.39 \%$ & $99.60 \%$ & $99.52 \%$ & $99.59 \%$ & $99.53 \%$ \\
Recall & $98.37 \%$ & $98.17 \%$ & $98.63 \%$ & $98.43 \%$ & $98.33 \%$ & $98.39 \%$ \\
F-measure & $98.96 \%$ & $98.77 \%$ & $99.11 \%$ & $98.98 \%$ & $98.95 \%$ & $98.96 \%$ \\
\hline
\end{tabular}

Table 4. Result of test set using 5-fold cross validation

\begin{tabular}{lllll}
\hline Dataset & Precision & Accuracy & Recall & F-measure \\
\hline $\mathrm{D}_{\text {test }}$ & $97.22 . \%$ & $99.73 \%$ & $97.46 \%$ & $98.59 \%$ \\
\hline
\end{tabular}

Table 5. Comparison of the method

\begin{tabular}{c|c|c|c}
\hline Method & total number & correct & incorrect \\
\hline $\begin{array}{c}\text { sliding window } \\
\text { the fetal heart cycle recognition } \\
\text { model }\end{array}$ & 1498 & 1373 & 125 \\
& 1509 & 1452 & 57
\end{tabular}

A new test evaluation of the model is performed using the test sample $\left(\mathrm{D}_{\text {test }}\right)$. The test results are shown in Table 4. It can be seen from the table that the recognition model performs well on the test sample. We also carried out a comparison with traditional method, and the results are shown in Table 5. The experiment result shows that more fetal heart rate can be extracted by the model of fetal heart cycle recognition and the accuracy rate is higher $(1452 / 1509)$.

Among the actual extraction of the test data, the model outputs 43 negative cases of fetal heart rate, of which 32 negative cases are correct, and 11 negative cases are misjudged after manual review. Among the 11 negative examples, 8 negative cases are slight misjudgments. As shown in Figure 5, the first extreme point of the AMDF curve can be discriminated ambiguously (as shown in the red box), but the periodic points of the envelope curve and the AMDF curve are not clear, which is a difficult situation to judge. In the future, we will expand and improve the training set to obtain better recognition results. Finally, in this paper, we make a statistical analysis about the detection of the whole section of the signal, the detection rate is $87.94 \%$ (excluding the worst part of the signal). Except for a few extraction error, other conditions in which the fetal heart rate could not be detected were due to the presence of large unstable factors or noises in local signals. In general, the detection has achieved ideal effect.

In general, the model can identify cycle points effectively, exclude the wrong cycle points and improve the accuracy of fetal heart rate extraction. 

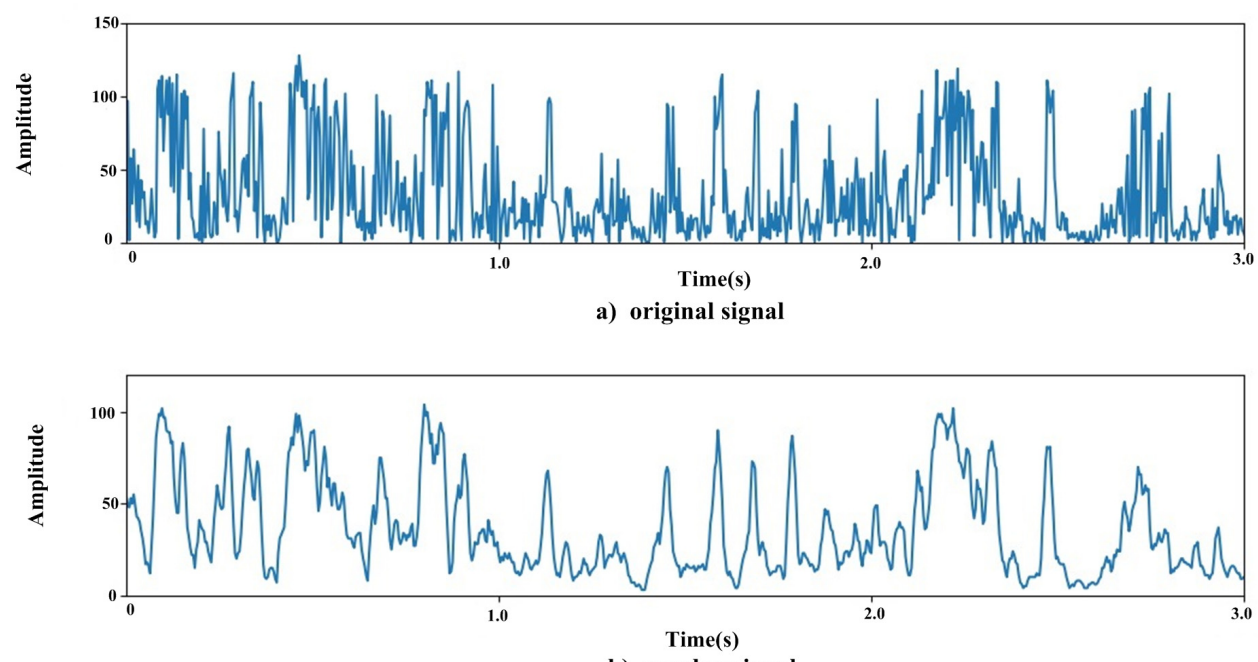

b) envelop signal

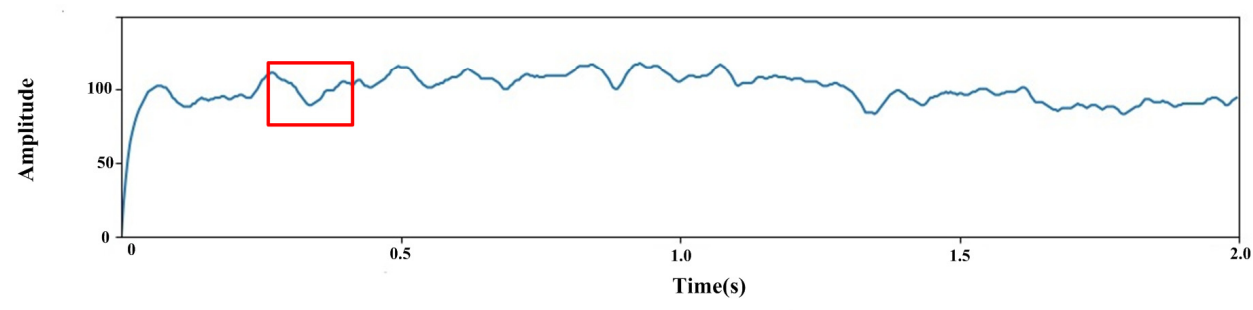

c) AMDF signal

Figure 5. A situation that the model output negative result

Based on the research and clinical research of related literature, this paper analyzed the limitations of the periodic point detection of autocorrelation algorithms used in the current fetal heart rate extraction. Given the situation that the fetal heart rate may be doubled or reduced or difficult to be identified, this paper proposed a model to assist in recognizing the fetal heart rate cycle based on ensemble algorithm combined with the characteristics of envelope curve and AMDF curve. In this model, multiple features of envelope curve and AMDF curve are used to assist the judgment of the identified periodic points, which improve the accuracy of fetal heart rate extraction.

In this paper, the data is inadequate. In the part of FHR extraction, although the training sample size reaches the level of nearly 100,000 through upsampling, the actual number of negative samples is still relatively insufficient. As the hospital can not provide fetal ECG signal as a reference standard ( because it is costly and easy to have adverse effects on pregnant women), the Doppler fetal heart sound signal used in this paper needs a manual comparison to determine the correct fetal heart rate label during the experiment. The workload is huge and there may be some manual errors. More investment are needed to obtain more samples.

As the focus of this research is feature extraction, in this paper, the filtering method used in the signal preprocessing is simple. In fact, other scholars have adopted a more complex filtering effect which has achieved good results. The model proposed in this paper can also be applied on the basis of these new preprocessing methods. Therefore, it is necessary to adopt interesting filtering method to improve the model in the future work.

For the model, it is simple to extract features from AMDF curve and envelope curve. In fact, some features in the AMDF curve are difficult to describe and extract clearly with rules. Deep learning can be used for features extraction and recognition, when the sample size is adequate.

\section{Conclusion}

CTG(Cardiotocogram) data is ubiquitously used for obstetricians to evaluate the well-being of fetuses before delivery. In this paper, we study a new method to extract FHR based on autocorrelation function. In the basic of 
AMDF, combining with envelope curve, we have developed a recognition model based on ensemble learning to assist in identifying FHR cycle. We have evaluated the performance of the model using three different performance measures, namely Precision, Recall and F-measure. In this paper, the result is compared with sliding window, and it can be seen that overall accuracy of the new method (96.2\%) is better than traditional method (91.7\%), which has enhanced the reliability and robustness of the fetal well-being monitoring, it can be the first step for further detection of fetal risk in pregnancy and toward a prediction of fetal outcome.

\section{Acknowledgments}

This work was supported by Science and Technology Planning Project of Guangdong Province, China.

\section{References}

Al-Angari, H. M., Kimura, Y., Hadjileontiadis, L. J. \& Khandoker, A. H. (2017). A hybrid EMD-kurtosis method for estimating fetal heart rate from continuous Doppler signals. https://doi.org/10.3389/fphys.2017.00641.

Chawla, N. V., Bowyer, K. W., Hall, L. O. \& Kegelmeyer, W. P. (2002). SMOTE: synthetic minority over-sampling technique. https://doi.org/10.1613/jair.953.

Hua, X., Kaiqing, L. \& Zhenxi, Z. (2005). A new algorithm for detecting fetal heart rate using ultrasound Doppler signals. https://doi.org/10.1016/j.ultras.2004.11.005

Jezewski, J., Roj, D., Wrobel, J. \& Horoba, K. (2011). A novel technique for fetal heart rate estimation from Doppler ultrasound signal. https://doi.org/10.1186/1475-925X-10-92.

Ko, S. I. (2018). U.S. Patent Application No. 15/774,679.

Lee, C. S., Masek, M., Lam, C. P. \& Tan, K. T. (2009, January). Towards higher accuracy and better noise-tolerance for fetal heart rate monitoring using Doppler ultrasound. https://doi.org/10.1109/TENCON.2009.5395885.

Marzbanrad, F., Kimura, Y., Funamoto, K., Sugibayashi, R., Endo, M., Ito, T., ... \& Khandoker, A. H. (2013). Automated estimation of fetal cardiac timing events from doppler ultrasound signal using hybrid models. https://doi.org/10.1109/JBHI.2013.2286155.

Peters, C. H. L., Broeke, E. D. M. T., Andriessen, P., Vermeulen, B., Berendsen, R. C. M. \& Wijn, P. $\quad$ F. $\quad$ F. (2004). Beat-to-beat detection of fetal heart rate: Doppler ultrasound cardiotocography compared to direct ECG cardiotocography in time and frequency domain. https://doi.org/10.1088/0967-3334/25/2/015

Peters, M., Crowe, J., Piéri, J. F., Quartero, H., Hayes-Gill, B., James, D \& Shakespeare, S. (2001). Monitoring the fetal heart non-invasively: a review of methods. https://doi.org/10.1515/JPM.2001.057

Voicu, I., Girault, J. M., Roussel, C., Decock, A. \& Kouame, D. (2010). Robust estimation of fetal heart rate from US Doppler signals. https://doi.org/10.1016/j.phpro.2010.01.087.

Weihong Cheng. \& Zhihou Cheng. (2018). Electronic fetal heart rate monitoring (2nd ed.).

Wong-Lam, H. W. \& Naley, M. (2001). A robust and accurate algorithm for time measurements of periodic signals based on correlation techniques. https://doi.org/10.1109/19.963181

Xiao Li. (2015). New Methods of Detection and Analysis of Fetal Heart Rate Electronic Monitoring[D].

\section{Copyrights}

Copyright for this article is retained by the author(s), with first publication rights granted to the journal.

This is an open-access article distributed under the terms and conditions of the Creative Commons Attribution license (http://creativecommons.org/licenses/by/3.0/). 